\title{
INTEGRAL REPRESENTATION OF SCHLÖMILCH SERIES
}

\author{
DRAGANA JANKOV AND TIBOR K. POGÁNY
}

\begin{abstract}
Certain integral representations are derived for the Schlömilch series of Bessel functions of the first kind $J_{v}$, using newly derived integral representation of first kind Kapteyn-type series.
\end{abstract}

Mathematics subject classification (2010): Primary: 40C10, 33C10.

Keywords and phrases: Schlömilch series, Kapteyn series, Bessel functions of the first and second kind, nonhomogeneous Bessel differential equation, Dirichlet series, Laplace integral expression of Dirichlet series.

\section{REFERENCES}

[1] Á. BARICZ, D. JANKov, T. K. PogÁNy, Integral representation of first kind Kapteyn series, J. Math. Phys. 52, 4 (2011), 043518-1-043518-7.

[2] V. F. BONDARENKO, Efficient summation of Schlömilch series of cylindrical functions, Comput. Math. Math. Phys. 31, 7 (1991), 101-104 (1992).

[3] C. V. CoAtes, Bessel's functions of the Second order, Quarterly Journal XXI (1886), 183-192.

[4] L. N. G. FILON, On the expansion of polynomials in series of functions, Proc. London Math. Soc. (2) IV (1906), 396-430.

[5] I. S. Gradshteyn, I. M. Ryzhik, Table of Integrals, Series, and Products, Sixth Ed., Academic Press, San Diego, CA, 2000.

[6] J. Karamata, Theory and Application of the Stieltjes Integral, Srpska Akademija Nauka, Posebna izdanja CLIV, Matematički institut, Knjiga I, Beograd, 1949. (in Serbian)

[7] L. Landau, Monotonicity and bounds on Bessel functions, in Proceedings of the Symposium on Mathematical Physics and Quantum Field Theory, Berkeley, California, 2000, 147-154; Electronic J. Differential Equations 4 (2002), Southwest Texas State University, San Marcos, Texas.

[8] A. R. Miller, m-dimensional Schlömilch series, Canad. Math. Bull. 38, 3 (1995), 347-351.

[9] N. Nielsen, Sur le développement de zéro en fonctions cylindriques, Math. Ann. LII (1899), 582587.

[10] N. NiELSEN, Flertydige Udviklinger efter Cylinderfunktioner, Nyt Tidsskrift X B (1899), 73-81.

[11] N. NiELSEN, Note sur les développements schloemilchiens en série de fonctions cylindriques, Oversigt K. Danske Videnskabernes Selskabs (1899), 661-665.

[12] N. NiELSEN, Note supplémentaire relative aux développements schloemilchiens en série de fonctions cylindriques, Oversigt K. Danske Videnskabernes Selskabs (1900), 55-60.

[13] N. Nielsen, Sur une classe de séries infinies analogues á celles de Schlömilch selon les fonctions cylindriques, Ann. di Mat. (3) VI (1901), 301-329.

[14] N. NIELSEN, Recherches sur une classe de séries infinies analogue á celle de M. W. Kapteyn, Oversigt K. Danske Videnskabernes Selskabs (1901), 127-146.

[15] N. NiELSEN, Handbuch der Theorie der Cylinderfenktionen, Leipzig, 1904.

[16] T. K. PogÁnY, E. SÜLI, Integral representation for Neumann series of Bessel functions, Proc. Amer. Math. Soc. 137, 7 (2009), 2363-2368.

[17] J. W. S. RaYleigh, On a Physical Interpretation of Schlömilch's Theorem in Bessel's Functions, Phil. Mag. 6 XXI (1911), 567-571.

[18] O.X. SChlÖmILCH, Note sur la variation des constantes arbitraires d'une intégrale définie, Journal für Math. XXXIII (1846), 268-280. 
[19] V. TwERS Ky, Elementary function representations of Schlömilch series, Arch. Rational Mech. Anal. 8 (1961), 323-332.

[20] G. N. Watson, A Treatise on the Theory of Bessel Functions, Cambridge University Press, Cambridge, 1922. 